\title{
Méthodes expérimentales pour l'étude de la dynamique des clapets flottants : compresseurs à pistons
}

\author{
Jean-Luc Dion ${ }^{\mathrm{a}}$ et Serge Vialard \\ ISMCM-CESTI, 3 rue Fernand Hainaut, 93407 Saint-Ouen Cedex, France
}

Reçu le 30 janvier 2002, accepté le 23 octobre 2003

\begin{abstract}
Résumé - La modélisation paramétrique du comportement dynamique des clapets de compresseurs à pistons nécessite un choix judicieux des paramètres sur lesquels le recalage est efficace. Cet article s'attache à décrire des expériences réalisées en vue de l'obtention de grandeurs propres à vérifier le bien fondé des modèles aussi bien que de permettre la résolution du problème inverse de la dynamique des clapets. L'analyse des résultats conduit à des modèles qui constituent des aides à la conception des clapets pour une optimisation des rendements mécanique, volumétrique et énergétique, donc aussi une réduction des vibrations, des chocs et du bruit.
\end{abstract}

Mots clés : Compresseur à pistons / clapet / écoulement pulsé / méthodes expérimentales

\begin{abstract}
Experimental methods for the valve dynamic study: reciprocating compressor. When modelling the dynamic behaviour of reciprocating compressor's valve, a sensible choice of parameters is necessary with a view to operate an effective calibration. This paper describes experiments realised in order to obtain parameters which are able, on one hand to verify the validity of models, and on the other hand to access to the resolution of the inverse problem for the valve dynamic. Results analysis leads to models that are good help for valve design with an objective of optimisation of mechanical, volumetric and energetic efficiency, which consequently leads to the reduction of the vibrations, impacts and noise.
\end{abstract}

Key words: Reciprocating compressor / valve / pulsed flow / experimental methods

\section{Introduction}

Les clapets flottants déclinés sous différentes solutions technologiques sont classiquement utilisés dans les compresseurs pneumatiques (Fig. 1) et frigorifiques. Ces clapets assurent les fonctions d'admission et de refoulement des compresseurs. En fonctionnement normal, les clapets subissent des ouvertures-fermetures plusieurs dizaines de fois par seconde. Ces ouvertures et fermetures sont provoquées par des différences de pression appliquées entre les deux faces d'un même clapet. Les phénomènes dynamiques (souhaités et indésirables) des clapets flottants conditionnent fortement le comportement global du compresseur. Les rendements mécanique, volumétrique et thermodynamique y sont étroitement liés. Les clapets jouent également un rôle déterminant dans le comportement acoustique du compresseur et sont des composants dont la durée de vie conditionne celle du compresseur.

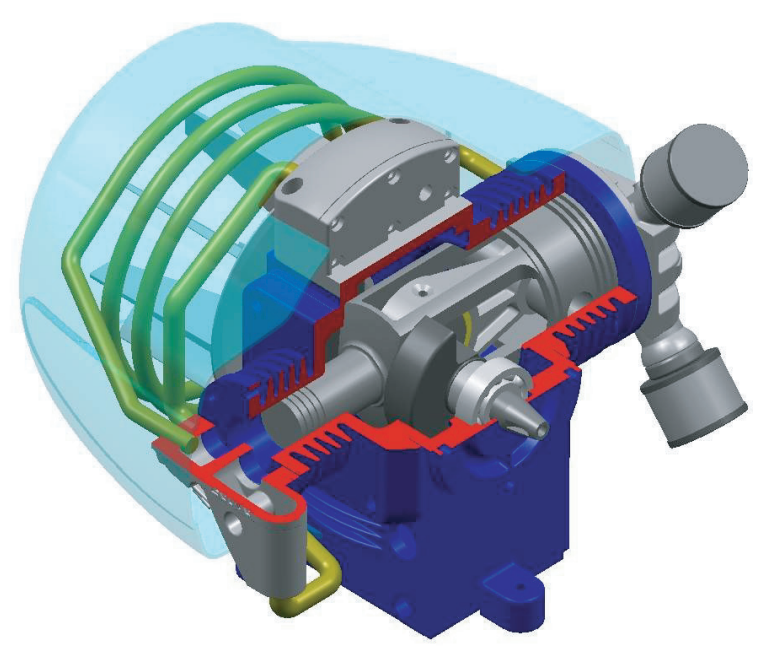

Fig. 1. Exemple de compresseur pneumatique.

a Auteur correspondant : dion@ismcm-cesti.fr 
Pour toutes ces raisons l'étude expérimentale et la compréhension des comportements dynamiques des clapets présentent un intérêt aussi bien scientifique qu'industriel.

\section{Objectifs et méthodes expérimentales}

Les expériences réalisées sont étroitement liées aux modèles retenus pour décrire les comportements dynamique des clapets : le choix des modèles est déterminé en fonction de la possibilité d'identifier leurs paramètres par l'expérience; les conditions de faisabilité des mesures conditionnent fortement le choix des modèles retenus. Par ailleurs, le choix des expériences réalisées est fixé par leur aptitude à vérifier le bien fondé des hypothèses des modèles de même que la précision qu'elles assurent lors de la résolution du problème inverse et de l'identification paramétrique.

Cette stratégie de réalisation des expériences a été appliquée pour déterminer les modèles qui régissent :

1. la thermodynamique des phases de compression et de détente;

2. les pertes de charge de l'écoulement au clapet;

3. la dynamique du clapet en vol libre;

4. la dynamique lors de l'impact du clapet sur son siège ou sa butée.

\subsection{Mesures thermodynamiques}

Le cycle complet d'une chambre de compression est composé de 4 phases : compression, échappement, détente, admission (Fig. 7).

Les phases de compression et détente s'effectuent entièrement avec tous les clapets fermés (pas d'admission ni d'échappement). La durée de chacune de ces phases est de l'ordre de quelques millièmes de secondes : les techniques expérimentales actuelles ne permettent pas de quantifier les échanges de chaleur ni les évolutions de température sur des durées aussi courtes dans des milieux aussi confinés que la chambre de compression. Seules des mesures de températures moyennes sont accessibles : le cycle dynamique des températures ne peut être reconstruit qu'à partir de modèles reliant la température, la pression et le volume. Le modèle peut alors être recalé sur la valeur moyenne de la température par intégration numérique sur un cycle de l'évolution de la température déduite des mesures de pression et de volume.

Le volume instantané de la chambre de compression est défini par la position instantanée du piston. Il devrait pouvoir être estimé par la détermination de la position du piston à partir d'une référence prise sur le vilebrequin du compresseur. Cette référence est ici synchronisée sur la position du point mort haut du piston. C'est dire que la position instantanée du piston est alors supposée complètement définie par la vitesse de rotation (mesurée par un top tour) et la cinématique du système biellemanivelle. La validité de cette démarche suppose qu'il

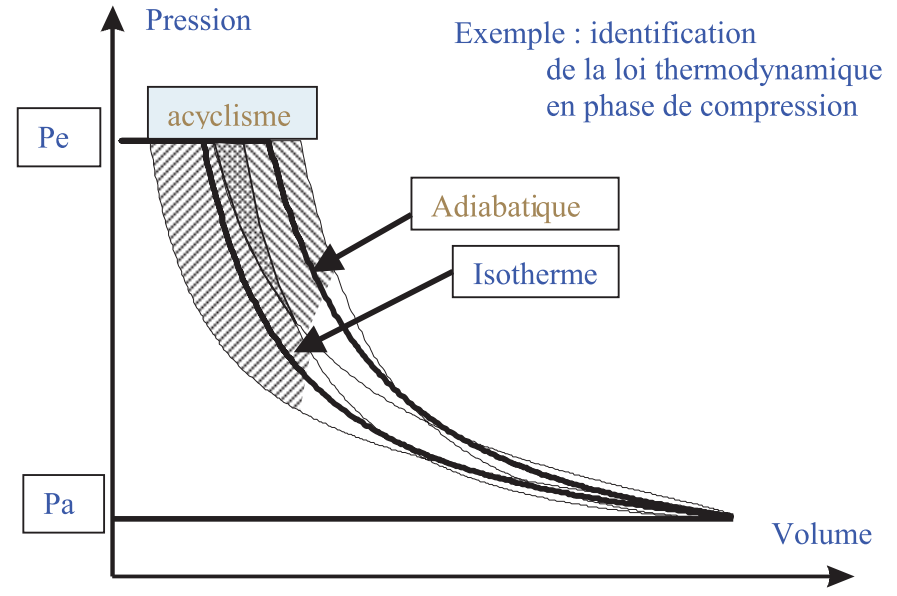

Fig. 2. Influence de l'acyclisme sur la phase de compression.

n'apparait pas d'irrégularités cycliques de la vitesse de rotation du vilebrequin, cette hypothèse n'est pas acceptable.

Dans le cas des compresseurs pneumatiques, le comportement thermodynamique des phases de compression et de détente se situe en réalité entre l'isotherme et l'adiabatique $(1<\gamma<1,4)$.

L'incertitude sur la position du point mort haut est de l'ordre du degré.

Les irrégularités cycliques (au cours d'un même cycle) sur la vitesse de rotation instantanée du vilebrequin peuvent être estimées à $\pm 5 \%$ de la vitesse moyenne de rotation du cycle dans certains cas.

Le calcul d'erreur issu de ces incertitudes de mesure met en évidence l'impossibilité d'identifier les lois de comportement thermodynamiques qui régissent les phases de compression et de détente sans tenir compte des irrégularités cycliques de vitesse (Fig. 2).

En effet, les zones grisées correspondent aux domaines d'incertitudes provoqués par les irrégularités cycliques de rotation du vilebrequin lors de la phase de compression. Les frontières de ces zones ont été obtenues à partir de relevés expérimentaux pour des taux de compression élevés qui favorisent les irrégularité cycliques. L'amplitude de ces domaines d'incertitude entache fortement la qualité des identifications paramétriques des lois de comportement thermodynamique. Certains paramètres très influents sur les performances du compresseur (coefficient «polytropique $» \gamma$, par exemple) peuvent alors être définis avec une incertitude de l'ordre de plusieurs dizaines de \% conduisant à une erreur systématique importante lors de l'utilisation du modèle. La mesure de vitesse de rotation instantanée au cours du cycle est indispensable pour l'identification de loi thermodynamique sur des compresseurs mono-piston. Pour les compresseurs multi-étagés (plusieurs pistons) le déphasage entre les pistons est souvent choisi de façon à répartir le plus uniformément possible les efforts sur le vilebrequin lors d'un cycle : les irrégularités cycliques sont alors beaucoup plus faibles. L'adjonction de volants d'inertie (ventilateur, accouplement, ... ) permet également une réduction sensible de 
ces irrégularités cycliques. Ces irrégularités cycliques sont également sources de consommation d'énergie importante pour les moteurs électriques d'entraînement des compresseurs. Le rendement énergétique global reste très sensible à ce paramètre.

L'identification des lois de comportement thermodynamique passe donc nécessairement par la connaissance de la vitesse de rotation instantanée du vilebrequin qui détermine le volume instantané dans la chambre de compression.

La mesure de la vitesse est réalisée grâce au signal d'une cellule photosensible placée en face d'un couronne solidaire du vilebrequin sur laquelle sont alternées des bandes sombres et réfléchissantes. Le signal est traité par démodulation de fréquence. La méthode retenue est basée sur la recomposition du signal analytique par transformée de Hilbert. Le signal analytique est multiplié par le signal de la porteuse afin d'obtenir la phase instantanée. Un filtrage numérique passe bas est réalisé pour des rapports faibles (inférieur à 20) entre la fréquence d'échantillonnage et la fréquence fondamentale du signal brut.

Le volume instantané de la chambre est déduit de la référence de phase (point mort haut du piston) et de la vitesse instantanée du vilebrequin.

La pression instantanée est mesurée directement dans la chambre de compression.

La relation pression-volume définie par la mesure peut alors être identifiée à des modèles thermodynamiques. Dans un deuxième temps, le choix du modèle thermodynamique permet de déduire la température instantanée du gaz dans la chambre de compression à partir des mesures précédentes.

\subsection{Pertes de charge de l'écoulement au clapet}

Les pertes de charge au clapet sont identifiées à partir de leur mesure en écoulement permanent et en écoulement pulsé. Les pertes de charge en écoulement pulsé sont mesurées dans les conditions de fonctionnement du compresseur.

Ces pertes de charge sont obtenues par la mesure des pressions dynamiques à l'amont et à l'aval du clapet qui doit être complétée par la mesure de la position instantanée du clapet afin d'identifier une loi de couplage fluidestructure. Le coefficient de perte de charge obtenu est supposé entièrement défini par les constantes géométriques du clapet et par sa position instantanée dans son environnement [1].

Les vitesses d'écoulement (ou débits) sont déduites des modèles d'écoulement et de couplage fluide-structure. Les débits instantanés ainsi obtenus sont recalés grâce à la mesure du débit moyen (la mesure du débit instantané étant inaccessible dans ce domaine de fréquence).

\subsection{Dynamique du clapet en vol libre}

La dynamique du clapet en vol libre et lors de ses impacts est mesurée directement par un capteur de

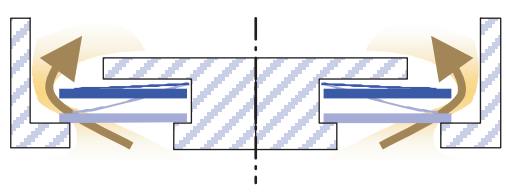

Fig. 3. Clapet annulaire.

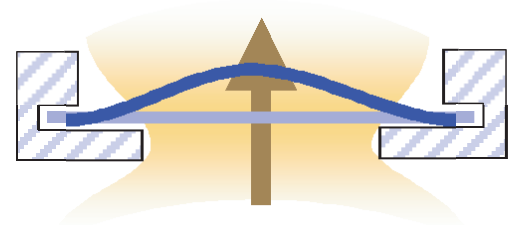

Fig. 4. Clapet à lamelle.

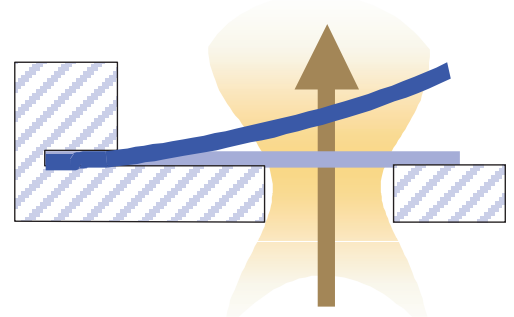

Fig. 5. Clapet « finger ».

déplacement sans contact. Les paramètres de rigidité et de masse du clapet sont identifiés par des mesures complémentaires hors compresseur. La masse est définie par mesure directe pour les clapets annulaires (Fig. 3), par recalage de la masse volumique pour les clapets « lamelles » (Fig. 4) ou « finger » (Fig. 5) lorsque la masse réelle diffère de la masse calculée par CAO. La rigidité des clapets annulaires est mesurée sur le ressort de clapet; le clapet est supposé de rigidité infinie devant le ressort de clapet. Les autres types de clapet ont une rigidité qui est recalée sur le module d'Young du matériau par identification des trois premières fréquences propres du clapet dans les conditions aux limites correspondant à son mode de fonctionnement. La mesure de la position instantanée du clapet en fonctionnement permet essentiellement de définir l'interaction fluide-structure lors des phases d'ouverture et de fermeture du clapet. En dehors de sa surface apparente devant l'écoulement, les paramètres caractéristiques du clapet peuvent être identifiés avec une précision satisfaisante par des modèles numériques en éléments finis.

\subsection{Dynamique lors de l'impact du clapet sur son siège ou sa butée}

La dynamique de l'impact reste de très loin la méthode entachée de la plus grande incertitude sur l'identification paramétrique des modèles.

Trois types de mesures sont mises en œuvre pour identifier les fonctions d'impact. Les mesures accélérométriques sur le corps du compresseur renseignent 


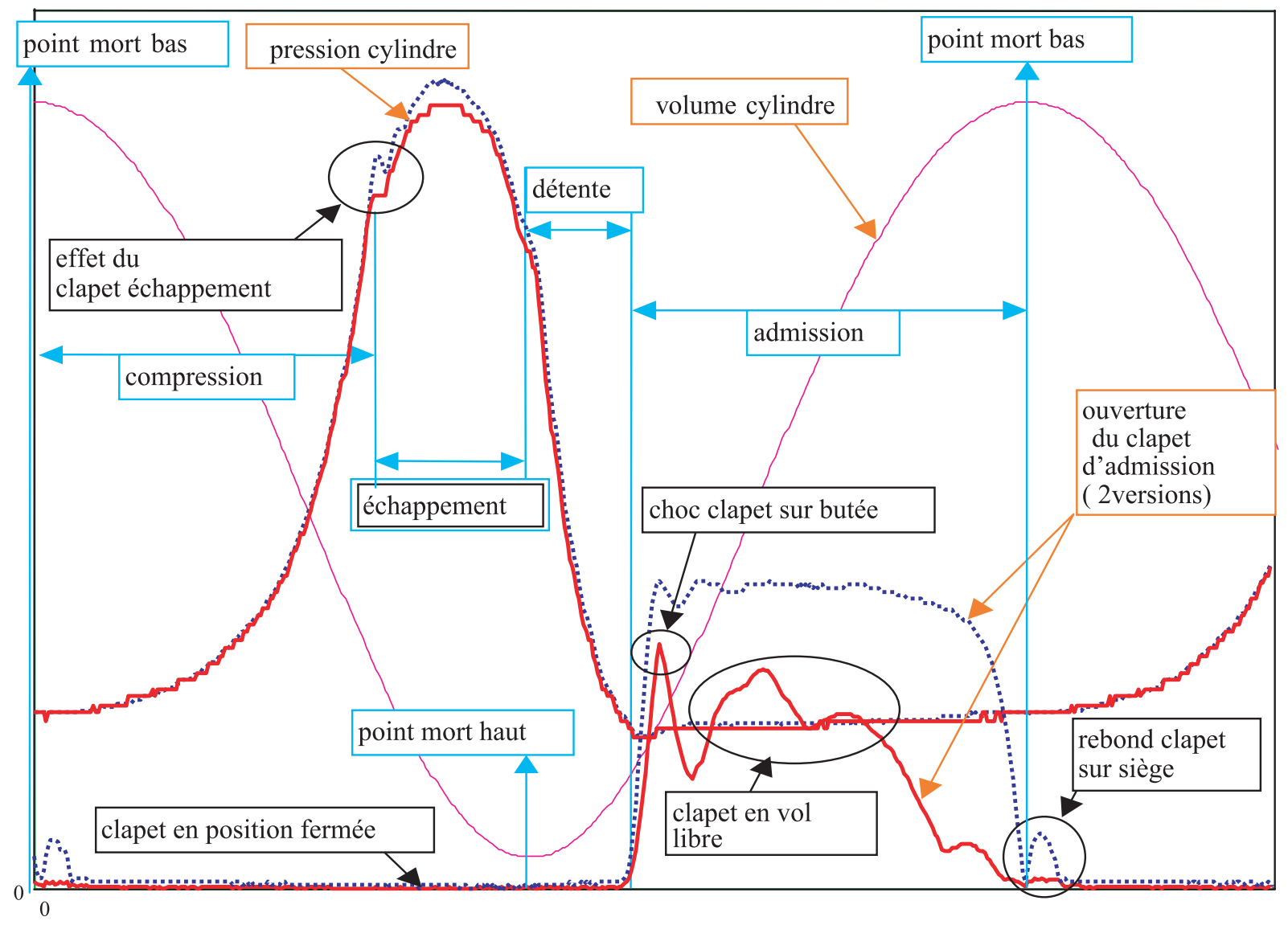

Fig. 6. Exemple de relevés expérimentaux.

qualitativement sur les impacts du clapet. La mesure du déplacement instantané conduit par dérivation à la connaissance des vitesses d'approche et de retour du clapet avant et après impact. Ces vitesses conduisent à l'identification des modèles basés sur un coefficient de restitution [2]. Toutefois, ces modèles ne permettent pas d'accéder correctement aux efforts et contraintes générés lors de l'impact.

Les modèles qui décrivent la dynamique de l'impact imposent d'identifier la rigidité dynamique (fortement non linéaire) entre les solides. Cette rigidité dynamique est définie partiellement par la géométrie et les matériaux des solides impactés mais dépend principalement des défauts géométriques des pièces en contact [3]. Bien que l'identification correcte de certains paramètres reste délicate, il apparaît que ceux-ci n'influencent pratiquement pas la modélisation globale. Des recalages sont actuellement nécessaires à partir des mesures réalisées en configuration de fonctionnement réel du compresseur afin d'identifier ce deuxième type de modèles.

\section{Résultats expérimentaux}

Les courbes de la figure 6 représentent différentes grandeurs physiques du fonctionnement du compresseur. Bien que présentant des unités différentes, elles sont synchronisées sur un même diagramme afin de mettre en évidence la chronologie des phénomènes étudiés. Deux essais sont superposées sur le diagramme (deux configurations de clapets ayant des caractéristiques différentes : courses, rigidités...). Ce type de diagramme permet de décrire précisément les quatre phases du cycle d'une chambre de compression. L'étude des chocs sur la butée ou sur le siège du clapet, son vol libre, ses retards à l'ouverture ou à la fermeture sont autant d'informations qui permettent de diagnostiquer des modes de fonctionnement et de recaler les modèles numériques lorsqu'ils sont utilisés à des fins prédictives. Ainsi, le modèle présente-t-il une aide à la conception des compresseurs en vue d'optimiser les rendements comme de limiter les vibrations et le bruit.

\section{Interprétations}

Parmi les différentes représentations qui conduisent à la compréhension des modes de fonctionnement d'un compresseur, le diagramme Pression-Volume est retenu pour cet exemple comme support dans le diagnostic et l'interprétation des phénomènes.

Sur le diagramme (Fig. 7) de l'évolution de la pression en fonction du volume, le cycle théorique est défini par le contour de la zone grisée. Ce cycle est entièrement déterminé par la pression d'admission $(\mathrm{Pa})$, la pression 


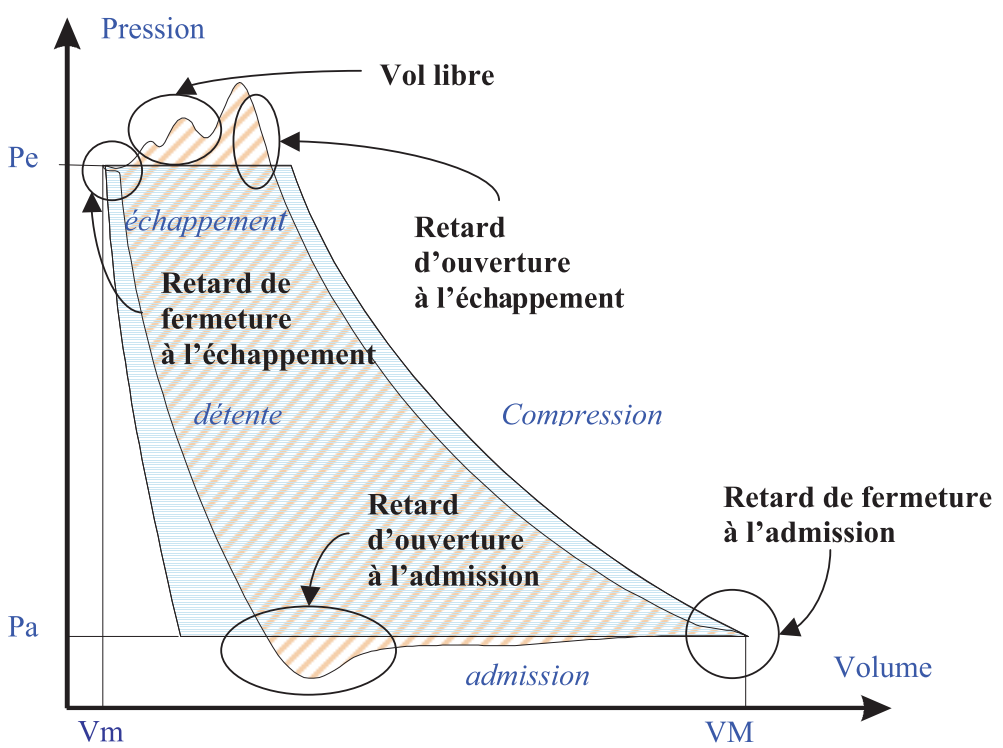

Fig. 7. Évolution de la pression en fonction du volume. d'échappement $(\mathrm{Pe})$, le volume mort ( Vm), le volume maximum de la chambre $(\mathrm{VM}=$ Volume engendré par le déplacement du piston + Volume mort) ainsi que la loi thermodynamique qui régit la détente et la compression.

Le cycle réel mesuré dans la chambre d'un compresseur pneumatique est représenté par le contour de la zone hachurée. Dans les deux cas la surface couverte correspond à l'énergie du cycle thermodynamique. Les différences entre les deux cycles portent sur quatre surfaces distinctes.

La surface « droite $»$ comprise entre les deux courbes de compression est due à un retard de fermeture des clapets d'admission. Le mouvement du piston amorce la réduction du volume de la chambre, le clapet d'admission n'étant pas encore fermé provoque sur un temps très court un phénomène de flux inverse (back-flow) : le clapet d'admission est en phase d'échappement. La surface couverte correspond à une énergie qui aurait dû être communiquée au fluide mais qui ne l'a pas été. Le phénomène affecte donc essentiellement le rendement volumétrique. Son existence n'est pas systématique : nombre de compresseurs possèdent une très bonne synchronisation entre le point mort bas du piston et la fermeture du clapet d'admission. Sauf erreur de conception des clapets d'admission, ce phénomène intervenant à basse pression (pression d'admission), il n'influence que très peu les rendements volumétrique et énergétique des compresseurs.

La surface « haute» comprise entre les courbes d'échappement théorique et expérimentale est dans un premier temps provoquée par un retard à l'ouverture du clapet d'échappement : le piston poursuit la phase de compression alors que la pression dans la chambre est supérieure à la pression d'échappement. Le retard à l'ouverture des clapets d'échappement peut être provoqué par des pertes de charges singulières ou une force de rappel des clapets (ressorts) trop importante mais dans la plupart des cas c'est la masse du clapet (forces inertielles) qui conditionne principalement ce retard. La surface couverte entre les deux courbes d'échappement correspond à une énergie dépensée mais non communiquée au fluide. Le rendement volumétrique n'est pas affecté par ce phénomène : seuls les rendements énergétiques s'en trouvent réduits. Le retard à l'ouverture se poursuit par un phénomène de vol libre du clapet d'échappement lors de son ouverture. Trois types de couplages conduisent à la dynamique du vol libre : le premier résulte d'une interaction entre l'écoulement du fluide et la position du clapet.

Le clapet est soumis à une différence de pression appliquée à la surface visible par le flux (la surface qui «obstrue » l'écoulement). Par ailleurs la position du clapet conditionne à tout instant la section offerte à l'écoulement (la surface « ouverte» au flux). Plus le clapet est fermé plus les pertes de charge singulières sont importantes : les forces d'ouvertures (différence de pressions) augmentent. Plus le clapet est ouvert, plus les pertes de charges sont faibles : le clapet tend alors à se refermer.

Le second couplage relie les caractéristiques de rigidité et de masse du clapet avec les sollicitations externes en pression et la position du clapet, il s'agit d'un couplage fluide structure « classique ».

Le troisième, plus rarement observé expérimentalement, couple les ondes de pression acoustique avec la position du clapet.

Les mouvements oscillants sans contact du clapet entre son siège et sa butée résultent de ces couplages physiques, ils définissent la phase de vol libre du clapet.

La surface « gauche» comprise entre les courbes de détente théorique et expérimentale est la conséquence d'un retard à la fermeture du clapet d'échappement : ce phénomène, comparable au retard à la fermeture du clapet d'admission, agit de façon beaucoup plus importante sur le rendement volumétrique : le volume balayé par le piston après dépassement du point mort haut et avant la fermeture du clapet d'échappement est rempli avec le gaz à la pression d'échappement. Le clapet d'échappement est en flux inverse : il est en phase admission. Le volume ainsi engendré s'ajoute au volume mort de la chambre. Ce gaz contenu dans le volume offert de la chambre doit être 
détendu de la pression d'échappement jusqu'à la pression d'admission avant que la phase d'admission puisse être déclenchée. Le gaz aspiré par le clapet d'échappement à la pression d'échappement va alors occuper un volume de l'ordre de dix fois supérieur lorsqu'il atteint la pression d'admission : le rendement volumétrique s'en trouve fortement réduit. En fonctionnement extrême, lorsque la proportion de gaz détendu devient trop importante par rapport à celle de gaz admis, un emballement thermique peut conduire à une ruine rapide du compresseur.

La surface «basse » est initiée par un retard à l'ouverture du clapet d'admission. Comme dans le cas d'un retard à l'ouverture du clapet d'échappement, la surface correspond à une énergie dépensée et non communiquée au fluide dans le bilan énergétique. Les niveaux de pressions sont sensiblement plus faibles que lors de la phase d'échappement, les phénomènes de vol libre s'avèrent souvent moins visibles sur le cycle thermodynamique.

\section{Conclusion et perspectives}

L'étude présentée s'attache à décrire exclusivement les aspect expérimentaux d'une étude conduite dans un objectif plus large sur la compréhension, la modélisation et la prédiction du fonctionnement des compresseurs. Les modèles exploités conjointement aux mesures réalisées ont permis d'atteindre les objectifs fixés (prévision des performances volumétrique, énergétique, thermique et vibratoire de nouveaux produits, mise au point de méthodes de dimensionnement des clapets). La réalisation des expériences est restée tout au long de cette étude étroitement liée au développement et à l'exploitation des modèles numériques. La compréhension des phénomènes mécaniques lors des impacts du clapet provient principalement des résultats de modèles et, à contrario, la description de certaines lois thermodynamiques reste principalement dictée par l'expérience dans une approche empirique des phénomènes (loi polytropique).

L'ensemble des mesures réalisées sur les compresseurs à piston a permis de mettre en évidence et de quantifier des dysfonctionnements, de minimiser les vibrations, les chocs et le bruit du compresseur, ce qui permet d'optimiser ses rendements mécanique, volumétrique et énergétique dés la conception.

Les travaux expérimentaux envisagés portent sur la mesure du comportement flexible des clapets en fonctionnement afin d'identifier les modèles de clapets flexibles. Cette information permettra un meilleur recalage des modèles éléments finis de type poutre et plaques actuellement utilisés pour décrire les clapets de type « finger » (Fig. 5) ou lamelle (Fig. 4) grâce à une analyse modale opérationnelle.

Remerciements. Les résultats expérimentaux de cette étude ont permis de recaler les modèles qui sont actuellement utilisés avec succès à des fin prédictives pour le dimensionnement des compresseurs à piston de la Société ERVOR.

\section{Références}

[1] I.E. Idel'cik, Memento des pertes de charge, Édition Eyrolles, 1986

[2] J.R. Lenz, Finite element analysis of dynamic flapper valve stresses, Fifteenth International Compressor Engineering Conference at Purdue University, West Lafayette, IN, USA, July 25-28, 2000

[3] F. Robbe-Valloire, R. Progri, B. Paffoni, R. Gras, Modélisation de la topologie microgéométrique, Matériaux et Techniques 3-4, 2000 